\title{
Self-Coexistence in the Dense Case for White Spaces
}

\author{
Julio Aráuz \\ J. Warren McClure School of ITS \\ Ohio University \\ Email: arauz@ohio.edu
}

\author{
Zachary Miller \\ J. Warren McClure School of ITS \\ Ohio University \\ Email: zm189308@ohio.edu
}

\begin{abstract}
In this paper we look at the downlink interference mitigation problem in 802.22 wireless regional area networks. We study the case where the density of TV bands is such that a group of white space base stations can obtain access to only one communications channel, a scenario likely to occur close to urban centers. We propose a self-organizing power control and design strategy to mitigate interference among base stations as well as an optimal approach for the problem.
\end{abstract}

\section{INTRODUCTION}

The secondary usage of TV or wireless microphone bands is what makes white spaces possible. The spectrum scarcity that plagues almost every broadband wireless access service is the main motivation behind the usage of these bands. However, the spectrum availability in these bands may widely vary depending on the proximity to incumbent users [1][2]. In our work we are interested in looking at the downlink in the dense case of white spaces deployment. We define this case as the scenario where television band devices (TVBDs) providing coverage to a particular area are able to access just one free channel. Previous work has estimated this may be common close to large metropolitan areas [1]. We study this problem under the framework provided by the IEEE 802.22 standard for wireless regional area networks (WRAN). To the best of our knowledge this case has not been looked at in the past.

The body of knowledge for interference management in infrastructure wireless networks is vast. Numerous optimization techniques have been proposed in the realms of linear and non linear programming. Usually these optimization approaches have as goal guaranteeing coverage or increasing throughput. On the other hand, interference management in an 802.22 network, where fixed TVBD provide coverage over a certain area to user devices, is closely related to recent research in the femtocell networks [3][4]. In general these approaches assume availability of interference information not present in 802.22. Moreover, in the power control area for white spaces, an interesting approach to protect primary incumbent users from TVBD interference was presented in [5]. In this work the authors proposed a constrained optimization problem to enhance the geo-location capabilities of a white space database in a way that throughput is maximized.

Due to editorial space limitations we will limit our review of related published material and rather place emphasis on our proposal. We believe that the foundations presented in

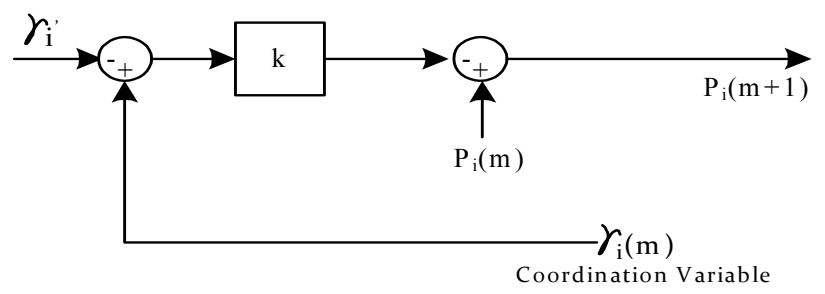

Fig. 1: Controller Structure at $\mathrm{BS}_{i}(i=1,2, \ldots, n ; m=0,1,2, \ldots)$ Control law: $P_{i}(m+1)=P_{i}(m)-k \times\left(\gamma_{i}(m)-\gamma_{i}^{\prime}\right)$, Gain: $k$

this article will enable interested readers to fully explore the benefits of our methodology, applied distributed control. Furthermore, we encourage interested readers to refer to [6] for further peer-reviewed material from the authors.

\section{BACKGROUND ON IEEE 802.22}

In an 802.22 network [7], base stations (BSs) provide wireless coverage to customer premises equipment (CPE) using unoccupied TV bands with cells that have a radius in the order of several tens of kilometers. In the dense case for white spaces there is only one band available. In this case a feature in 802.22 that is vital to maintain operation is referred to as self-coexistence. In self-coexistence mode, base stations with overlapping coverage using the same channel share the spectrum on a per frame basis. In this mode, each base station is autonomously allocated a subset of frames from a 16 frame superframe structure. Then each BS and the CPEs in a WRAN cell transmit only during their active frame(s) allocated in the superframe. A BS goes into self-coexistence operation when it cannot acquire an empty channel after initialization. In such a situation the BS is forced to select a channel already occupied by one or more WRAN cells and needs to identify if the interference comes from BSs or CPEs in other cells. To enable assignment of frames in a super frame to the BSs in overlapping areas, 802.22 introduces an On-demand Frame Contention mechanism. This mechanism is based on the exchange of messages between overlapping BSs to resolve the number and location of the frames to be shared during selfcoexistence.

\section{Self-Organizing Control Strategy}

In our study we focus on mitigating the downlink interference among BSs. We iteratively compute the transmission 
power of each BS, $P_{i}(i=1,2, \ldots, n)$, using a controller with the structure shown in Figure 1. The controller we employ has a structure similar to that of a proportional derivative (PD) one; it controls the output power of a BS at each iteration $m$. $\gamma_{i}(m)$ is our coordination variable which is fed back to the controller and defined as the average of the SINR values reported by a subset of the CPEs to base station $i$ in iteration $m(m=0,1,2, \ldots)$. We define $\gamma_{i}^{\prime}$ as the target value the coordination variable $\gamma_{i}$ should iteratively acquire in the long term.

\section{A. Controller Design}

The controller design is based on the selection of which CPEs will be included in the computation of $\gamma_{i}$ at BS $i$. Each BS at initialization receives SINR reports from the CPEs in the area and constructs a report vector $\mathbf{R}=\left[r_{i j}\right](i=$ $1,2, \ldots, n)(j=1,2, \ldots, q)$. Where $r_{i j}$ is the SINR in the downlink measured by CPE $j$ from BS $i$. Then each BS computes $v_{i}$ the average of the SINR reported from the CPEs in $\mathbf{R}$ and shares this parameter through the backhaul with all other BSs. After receiving all $v_{i}$ values each BS can locally construct vector $\mathbf{V}=\left[v_{i}\right](i=1,2, \ldots, n)$ and compute $\Theta$ as the average value of the elements in $\mathbf{V}$.

The scalar $\Theta$ is used to create a heuristic based association between BS $i$ and the CPEs that will contribute to its coordination variable $\gamma_{i}$. Base station $i$ selects for computation of $\gamma_{i}$ only those SINR reports from CPEs that at initialization have reported an SINR value greater than or equal to $\Theta$. This strategy in general allows BS $i$ to select as contributors to the feedback loop mainly those CPEs close to it. In the case a BS finds that no CPE has a reported SINR greater than or equal to $\Theta$ it does not participate in the cooperative control strategy. In this case the BS is probably located far away from regions of high densities of CPEs and a reduction of transmission power may hinder full coverage of a region.

Once all BSs have selected the CPEs whose SINR reports will contribute to their local coordination variable $\gamma_{i}$ the control loops adjust power accordingly to reach the individual goals. The control strategy proposed is simple and robust but it places stress on signaling necessary in the backhaul as every CPE needs to report the values $r_{i j}$ in each iteration. We stop the control loops after $\left|\gamma_{i}(m)-\gamma_{i}^{\prime}\right|=\varepsilon(\varepsilon \rightarrow 0)$, for all BSs. Finally, after the goals are reached we check if there are still overlapping areas between the BSs by looking at the SINR reports from all CPEs.

The controller depicted in Figure 1 also requires selecting a value for the proportional gain $k$. This gain allows tuning how radically a BS adjusts its transmission power in each iteration. We will use a heuristic approach to assign a gain value.

\section{B. Optimal solution}

To compare our control theory based approach with a optimal baseline we propose a mixed integer linear program that minimizes the number of BSs that a CPE receives service from. To formulate the program for a total of $n$ BSs and $q$ CPEs, consider the binary variable $y_{i j}$ as:

$$
y_{i j}= \begin{cases}1, & \text { if BS } i \text { covers CPE } j \\ 0, & \text { otherwise }\end{cases}
$$

The program's goal via the objective function is to minimize the number of CPEs covered by multiple BSs, therefore:

Minimize

$$
\sum_{i=1}^{n} \sum_{j=1}^{q} y_{i j}
$$

Subject to:

$$
\begin{gathered}
\alpha_{i j} \times P_{i} \geq \delta y_{i j}, \text { for } i=1,2, \ldots, n \text { and } j=1,2, \ldots, q \\
\sum_{i=1}^{n} y_{i j} \geq 1, \quad \text { for } j=1,2, \ldots, q \\
P_{i} \leq P_{\text {max }}, \quad \text { for } i=1,2, \ldots, n
\end{gathered}
$$

Coverage is considered in constraint (2). The transmission power of $\mathrm{BS}_{i}$ is represented by $P_{i}$ and the channel losses between $\mathrm{BS}_{i}$ and $\mathrm{CPE}_{j}$ by $\alpha_{i j}$. With constraint (3) we require any $\mathrm{CPE}_{j}$ to be covered by at least one BS. Constraint (4) limits the maximum power any BS can select. We recognize that the proposed optimum solution is a variation of the set covering problem and thus is not scalable with the number of CPEs and BSs.

\section{System Performance}

\section{A. Simulation Framework}

We evaluate the performance of the proposed solutions via simulation. In our study, $n$ BSs are used to cover a given geographical area and have access to just one unoccupied TV channel of $6 \mathrm{MHz}$. Table I lists all the parameters and the levels chosen for the study.

TABLE I: Simulation Study Parameters

\begin{tabular}{ll}
\hline \hline Parameter & Level \\
\hline Study area & $10 \mathrm{Km} \times 10 \mathrm{Km}$ \\
Carrier frequency $(1 / \lambda)$ & $400 \mathrm{MHz}$ \\
Max BS transmission power & $36 \mathrm{dBm}$ \\
BS antenna height $\left(h_{t x}\right)$, gain & $15 \mathrm{~m}, 12 \mathrm{~dB}$ \\
CPE antenna height $\left(h_{r x}\right)$, gain & $10 \mathrm{~m}, 9 \mathrm{~dB}$ \\
CPE sensitivity & $-110 \mathrm{dBm}$ \\
Noise figure, runs per experiment, $\varepsilon$ & $5 \mathrm{~dB}, 10,0.001$ \\
Number of BSs $(n)$, CPEs $(q)$ & $\{3,4,5\},\{5,10,15,20,25,30\}$ \\
BS locations & $1 \mathrm{Km}$ from corners of study area \\
CPE locations & Uniformly distributed $[1 \mathrm{~m}, 1 \mathrm{Km}]$ \\
\hline
\end{tabular}

To evaluate propagation losses we follow the model proposed in [8] specifically developed for white space analysis. We parametrize the model to match the propagation characteristics shown in Figure 3 of [8] for a carrier frequency of 400 MHz. Without loss of generality we selected a typical design based coverage value of $12 \mathrm{~dB}$ for $\gamma_{i}^{\prime}, \forall i$. 


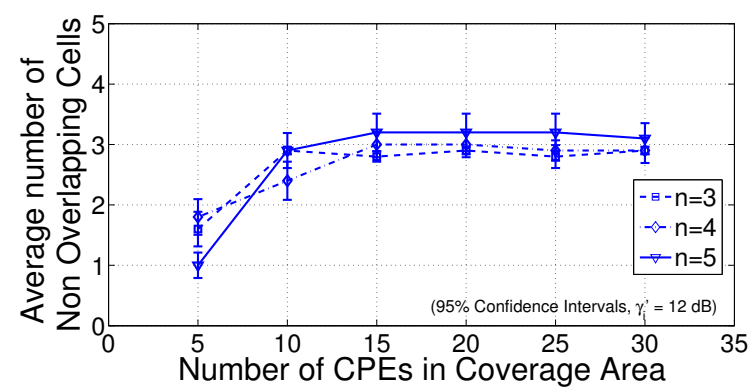

Fig. 2: Average Number of Non Overlapping Cells Cooperative Control Strategy

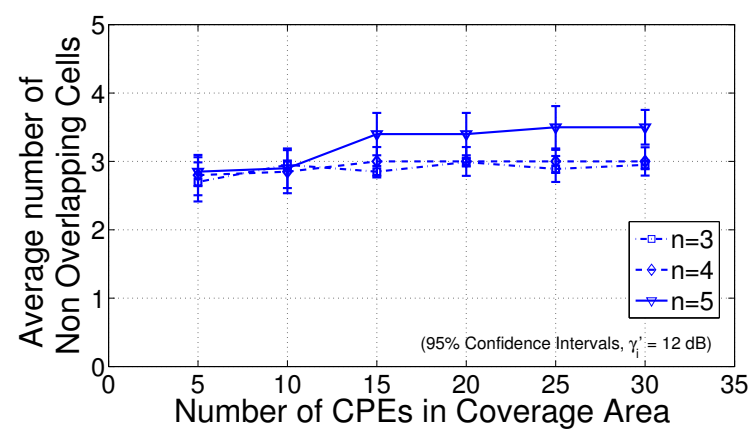

Fig. 3: Average Number of Non Overlapping Cells Optimal Solution

\section{B. Results}

We studied the performance of the system by looking at its ability to autonomously find power allocations that result in lowering the number of overlapping WRAN cells. All experiments started with the BSs employing their maximum transmission power for the initial calculation of feedback information. When a power allocation from the cooperative control strategy resulted in loss of coverage for a particular $\mathrm{CPE}$ we use the last power allocation before loss of service for the computation of the results.

From Figure 2 it is clear that as the number of CPEs in the coverage area increases it is easier to find power allocations that result in a larger number of non overlapping cells. Typically with a low number of CPEs, base stations do not have users that can be consistently grouped in regions close to them. Nevertheless, the average number of non

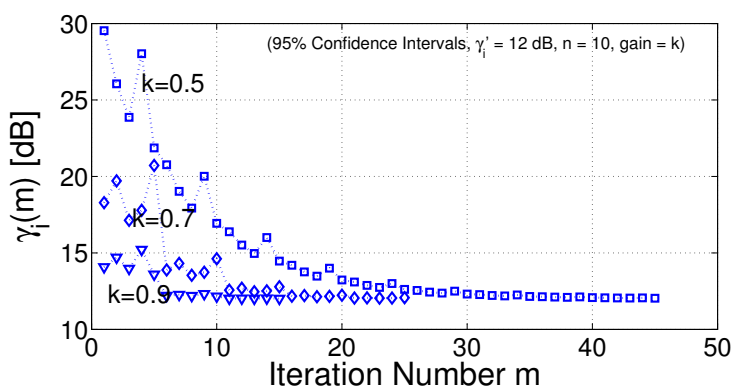

Fig. 4: Evolution Over Various Iteration of $\gamma_{i}$ for a Typical BS overlapping WRAN cells is between 1 and 2 for all three cases when there are only five CPEs in the area. This results in BSs needing to share a lower number of frames from the superframe among themselves and in some cases all BSs not having to share any bandwidth at all. As the number of CPEs increases the cooperative control strategy consistently finds an average number of non overlapping cells between 2 and 3.2. The performance of the optimal solution is portrayed in Figure 3. A careful comparison of the average values and confidence interval magnitudes between results indicates that the cooperative control strategy consistently yields results very close to the optimum (average difference among all cases of approximately $13 \%$ ) especially when a higher number of CPEs is present. We also explored the settling time of the value of $\gamma_{i}$ over a number of iterations. The results are presented in Figure 4 and further analysis showed that only 20 iterations are generally needed to achieve $95 \%$ of the goal.

\section{Closing Comments}

We looked at a simple, yet robust, strategy to find downlink power allocations for base stations in 802.22 networks. The results obtained indicated that a simple control rule can yield results that are close to the proposed optimum, while maintaining simplicity of implementation. Our study can be potentially extended to look at downlink throughput gains as the cooperative control strategy tends to group CPEs around the BS they receive at the highest power.

A concern in any closed loop solution is the amount of overhead. We believe that this will not be a critical factor that hinders implementability as once a solution is found there is no constant need to perform continuous updates unless conditions significantly change which is not likely in 802.22 networks. Furthermore, the system requires few iterations to stabilize. Nevertheless, we leave a detailed study on the impact on overhead and signaling as an option for future research.

\section{REFERENCES}

[1] K. Harrison, S. Mishra, and A. Sahai, "How Much White-Space Capacity Is There?" in New Frontiers in Dynamic Spectrum, 2010 IEEE Symposium on, April 2010, pp. $1-10$.

[2] "White space database," http://whitespaces.spectrumbridge.com/ whitespaces/home.aspx/, Accessed: August 01, 2012.

[3] M. Bennis and D. Niyato, "A Q-learning based approach to interference avoidance in self-organized femtocell networks," in GLOBECOM Workshops (GC Wkshps), 2010 IEEE, December 2010, pp. $706-710$.

[4] J. Aráuz, Z. Miller, and A. Sánchez, "Self-Organized Distributed Cooperative Control of Interference in Femtocell Networks," in iCOST '12. Proceedings of the 2012 International Conference on Selected Topics in Mobile and Wireless Networking, June 2012.

[5] S. Y. Lee, M. K. Kwon, and S. H. Lee, "Transmit power control scheme for tv white space wireless system," in Advanced Communication Technology (ICACT), 2011 13th International Conference on, Feb. 2011, pp. $1025-1029$.

[6] "White space analysis," http://its.ohiou.edu/arauz/docs/ws, Accessed: October $01,2012$.

[7] C. Stevenson, G. Chouinard, Z. Lei, W. Hu, S. Shellhammer, and W. Caldwell, "IEEE 802.22: The first cognitive radio wireless regional area network standard," Communications Magazine, IEEE, vol. 47, no. 1, pp. $130-138$, January 2009.

[8] D. Gurney, G. Buchwald, L. Ecklund, S. Kuffner, and J. Grosspietsch, in New Frontiers in Dynamic Spectrum Access Networks, 2008. DySPAN 2008. 3rd IEEE Symposium on, title=Geo-Location Database Techniques for Incumbent Protection in the TV White Space, Oct. 2008, pp. 1 -9. 